\title{
In-depth characterization of a long-term, resuscitated model of acute subdural hematoma-induced brain injury
}

\author{
Thomas Datzmann, MD, , 2 Thomas Kapapa, MD, PhD, ${ }^{3}$ Angelika Scheuerle, MD, ${ }^{4}$ \\ Oscar McCook, BSc, ${ }^{1}$ Tamara Merz, PhD, ${ }^{1}$ Sarah Unmuth, ${ }^{1}$ Andrea Hoffmann, MD, ${ }^{1}$ \\ René Mathieu, MD, ${ }^{5}$ Simon Mayer, MD, ${ }^{5}$ Uwe Max Mauer, MD, PhD, ${ }^{5}$ Stefan Röhrer, MD, ${ }^{6}$ \\ Deniz Yilmazer-Hanke, MD, PhD, ${ }^{7}$ Peter Möller, MD, PhD, ${ }^{8}$ Benedikt Lukas Nussbaum, MD, ${ }^{1,2}$ \\ Enrico Calzia, MD, PhD, ${ }^{1}$ Michael Gröger, PhD, ${ }^{1}$ Clair Hartmann, MD, ${ }^{1,2}$ \\ Peter Radermacher, MD, PhD, ${ }^{1}$ and Martin Wepler, MD ${ }^{1,2}$
}

${ }^{1}$ Institut für Anästhesiologische Pathophysiologie und Verfahrensentwicklung; ${ }^{2}$ Klinik für Anästhesiologie; ${ }^{3}$ Klinik für Neurochirurgie, ${ }^{4}$ Sektion Neuropathologie, Institut für Pathologie, ${ }^{7}$ Sektion Klinische Neuroanatomie, Klinik für Neurologie; and ${ }^{8}$ Institut für Pathologie, Universitätsklinikum, UIm; ${ }^{5}$ Klinik für Neurochirurgie, Bundeswehrkrankenhaus UIm; and ${ }^{6}$ Abteilung für Neurochirurgie, Klinikum Aalen, Germany

OBJECTIVE Acute subdural hematoma (ASDH) is a leading entity in brain injury. Rodent models mostly lack standard intensive care, while large animal models frequently are only short term. Therefore, the authors developed a long-term, resuscitated porcine model of $\mathrm{ASDH}$-induced brain injury and report their findings.

METHODS Anesthetized, mechanically ventilated, and instrumented pigs with human-like coagulation underwent subdural injection of $20 \mathrm{~mL}$ of autologous blood and subsequent observation for 54 hours. Continuous bilateral multimodal brain monitoring (intracranial pressure [ICP], cerebral perfusion pressure [CPP], partial pressure of oxygen in brain tissue $\left[\mathrm{PbtO}_{2}\right]$, and brain temperature) was combined with intermittent neurological assessment (veterinary modified Glasgow Coma Scale [MGCS]), microdialysis, and measurement of plasma protein S100ß, GFAP, neuron-specific enolase [NSE], nitrite+nitrate, and isoprostanes. Fluid resuscitation and continuous intravenous norepinephrine were targeted to maintain CPP at pre-ASDH levels. Immediately postmortem, the brains were taken for macroscopic and histological evaluation, immunohistochemical analysis for nitrotyrosine formation, albumin extravasation, NADPH oxidase 2 (NOX2) and GFAP expression, and quantification of tissue mitochondrial respiration.

RESULTS Nine of 11 pigs survived the complete observation period. While ICP significantly increased after ASDH induction, $\mathrm{CPP}, \mathrm{PbtO}_{2}$, and the MGCS score remained unaffected. Blood S100 $\mathrm{\beta}$ levels significantly fell over time, whereas GFAP, NSE, nitrite+nitrate, and isoprostane concentrations were unaltered. Immunohistochemistry showed nitrotyrosine formation, albumin extravasation, NOX2 expression, fibrillary astrogliosis, and microglial activation.

CONCLUSIONS The authors describe a clinically relevant, long-term, resuscitated porcine model of ASDH-induced brain injury. Despite the morphological injury, maintaining CPP and $\mathrm{PbtO}_{2}$ prevented serious neurological dysfunction. This model is suitable for studying therapeutic interventions during hemorrhage-induced acute brain injury with standard brain-targeted intensive care.

https://thejns.org/doi/abs/10.3171/2019.9.JNS191789

KEYWORDS GFAP; MAP-2; mitochondrial respiration; oxidative stress; multimodal brain monitoring; trauma

$\mathrm{A}$ CUTE subdural hematoma (ASDH) is frequent in traumatic brain injury, ${ }^{18}$ particularly in elderly patients ${ }^{23}$ and those with comorbidities, with mortality reaching $60 \% .^{44}$ Animal models of brain injury mostly use young and healthy rodents, which have a lissencephalic brain, ${ }^{10,45}$ and commonly lack standard intensive care. ${ }^{10}$
Large animals with gyrencephalic, human-like brains may improve clinical translation due to similar neuroanatomical structure. ${ }^{39}$ Standard clinical parameters including neurological assessment need to be integrated into translational research experiments. ${ }^{9}$ However, in addition to cost and necessary infrastructure, large animal models mostly

ABBREVIATIONS ASDH = acute subdural hematoma; $\mathrm{CPP}=$ cerebral perfusion pressure; ICP = intracranial pressure; I/E = inspiratory/expiratory; MGCS = modified Glasgow Coma Scale; NO = nitric oxide; NOX2 = NADPH oxidase 2; NSE = neuron-specific enolase; $\mathrm{PbtO}_{2}=$ partial pressure of oxygen in brain tissue; $\mathrm{PEEP}=$ positive end-expiratory pressure; ROS = reactive oxygen species.

SUBMITTED June 28, 2019. ACCEPTED September 13, 2019.

INCLUDE WHEN CITING Published online December 20, 2019; DOI: 10.3171/2019.9.JNS191789. 
comprise short observation periods ( $2-10$ hours only), and often lack intensive care $\mathrm{r}^{1-3,12,28,30,41,43}$ and/or a neurological assessment. ${ }^{10}$ Studying pathophysiological mechanisms and treatment approaches requires longer-term, largeanimal models with a well-reproducible type of injury and integration of current intensive care standards. Since ASDH is a well-reproducible model of acute brain injury, ${ }^{11}$ we developed a long-term, resuscitated model of ASDHinduced brain injury in human-sized pigs comprising bilateral, multimodal brain monitoring integrating standard intensive care, intermittent neurological assessment, measurement of blood biomarkers of brain injury as well as postmortem morphological and immunohistochemical analyses. ${ }^{5}$

\section{Methods}

After obtaining approval from the local Animal Care Committee and the Federal Authorities for Animal Research, we performed the experiments in adherence with the National Institutes of Health Guidelines on the Use of Laboratory Animals and the European Union "Directive 2010/63/EU on the protection of animals used for scientific purposes." Eleven pigs were used for this study; 2 animals had to be dropped from the study due to cardiovascular failure unrelated to the neurosurgical instrumentation or the ASDH induction, with high-dose norepinephrine needs $>4$ hours after ASDH induction, without any necropsy findings of herniation or neurosurgical instrumentation-related injuries. Hence, the data presented refer to 9 Bretoncelles-Meishan-Willebrand pigs of both sexes ( 5 females and 4 males; median age 11 months [IQR 9-12 months] and weight $65 \mathrm{~kg}$ [IQR 57-71 kg]). Coagulation properties in this pig strain closely mimic those in humans, ${ }^{31}$ in contrast to the otherwise species-specific hypercoagulability ${ }^{21}$ thereby mimicking the time needed for clot formation within the subdural space in humans.

During the last 12 hours preceding the experiments, the pigs received a nutritional solution (Fresubin, Fresenius Kabi) and had free access to water. Prior to induction of anesthesia, premedication consisted of intramuscular 5 $\mathrm{mg} / \mathrm{kg}$ azaperone and $1-2 \mathrm{mg} / \mathrm{kg}$ midazolam. After establishment of intravenous access via an ear vein, anesthesia was induced with propofol (1-2 mg/kg) and ketamine (1 $\mathrm{mg} / \mathrm{kg}) .{ }^{18,20}$ The pigs were endotracheally intubated and mechanically ventilated (ventilator settings: tidal volume $8 \mathrm{~mL} / \mathrm{kg}$, respiratory rate $8-12$ breaths/minute adapted to achieve a $\mathrm{PaCO}_{2}$ of 35-40 $\mathrm{mm} \mathrm{Hg}$, inspiratory/expiratory $[\mathrm{I} / \mathrm{E}]$ ratio of $1: 1.5$, fraction of inspiratory oxygen $\left[\mathrm{FiO}_{2}\right]$ ) of 0.3 , positive end-expiratory pressure [PEEP] $10 \mathrm{~cm}$ $\mathrm{H}_{2} \mathrm{O}$ to prevent formation of atelectasis). ${ }^{18,20}$ Anesthesia was maintained with continuous intravenous propofol (10 $\mathrm{mg} / \mathrm{kg} / \mathrm{hr}$ ) and fentanyl $(10 \mu \mathrm{g} / \mathrm{kg}$ initial bolus followed by $2.5 \mu \mathrm{g} / \mathrm{kg} / \mathrm{hr})$. A balanced electrolyte solution $(10 \mathrm{~mL} / \mathrm{kg} /$ hr, Jonosteril 1/1, Fresenius Kabi) was infused as maintenance fluid.

After surgical exposure, a 9-F catheter sheath was inserted into the right femoral vein for placement of a 3-lumen venous catheter (Arrow International) into the iliac vein. The right carotid artery was surgically exposed for placement of a precalibrated ultrasonic flow probe around the vessel. Both femoral arteries were exposed for placement of a 4-F PiCCO catheter (PULSION Medical Systems SE) for continuous cardiac output, pulse pressure variation measurement, and blood sampling. A catheter was placed in the urinary bladder via midline minilaparotomy.

The skull was exposed, and a craniotomy (approximately $20 \mathrm{~mm}$ ) was drilled over the left and right parietal cortices (Fig. 1). After exposing the dura on the left side, a small incision was made, and a catheter was inserted approximately $5 \mathrm{~mm}$ into the subdural space for later initiation of the ASDH. On the contralateral (right) side, an additional burr hole was similarly placed. Afterward, microdialysis catheters and multimodal brain monitoring probes (Neurovent-PTO, Raumedic AG) were inserted about $10-15 \mathrm{~mm}$ into the brain parenchyma in both hemispheres. The multimodal probes were placed under visual control for intracranial pressure (ICP), partial pressure of oxygen in brain tissue $\left(\mathrm{PbtO}_{2}\right)$, and temperature measurements. The probes were calibrated before insertion according to the manufacturer's specifications. After placement, all catheters were allowed to equilibrate for about 1 hour, and recording was started when $\mathrm{PbtO}_{2}$ values were stable. At the end of the neurosurgical procedure, the burr holes were closed using bone wax, which also served for fixation of the catheter as well as the microdialysis and ICP probes (Fig. 1). During surgery, hydroxyethyl starch $6 \% 130 / 0.42$ (Vitafusal, Serumwerk) was used to maintain pulse pressure variation, with a maximum dose of $30 \mathrm{~mL} /$ $\mathrm{kg}$ according to the manufacturer's specifications. The bilateral neurosurgical procedure was performed to avoid sham experiments, i.e., the hemisphere without ASDH served as the control for the hemisphere with ASDH, and complies with the $3 \mathrm{R}$ principle (replacement, reduction, and refinement of animal research). ${ }^{25}$

Figure 1 shows the experimental protocol. After neurosurgical instrumentation, in order to mimic a patient's situation immediately before the occurrence of ASDH, at least 1 hour before induction of $\mathrm{ASDH}, \mathrm{FiO}_{2}$ was reduced to 0.21 and the PEEP to $0 \mathrm{~cm} \mathrm{H}_{2} \mathrm{O}$, and the $\mathrm{I} / \mathrm{E}$ ratio was set to $1: 2$ for baseline measurements. Thereafter, $20 \mathrm{~mL}$ of autologous blood (corresponding to $12.5 \%$ of brain volume) was injected using an automated syringe pump (rate $90 \mathrm{~mL} / \mathrm{hr}$ to avoid clotting in the syringe; Perfusor Space, B. Braun Melsungen AG) via the subdural catheter above the left parietal cortex. This amount of blood was injected based on that in previous studies on porcine $\mathrm{ASDH}^{30,38,41}$ that had also investigated the effects of blood volumes corresponding to $10 \%-15 \%$ of the brain volume. Figure 2 shows a typical time course of ICP during and until the first 30 minutes after ASDH induction. Two hours after subdural blood injection, resuscitation was initiated: cerebral perfusion pressure $(\mathrm{CPP}=$ mean arterial pressure - ICP) was titrated to baseline levels; if CPP remained below baseline values despite volume resuscitation, norepinephrine was used to restore CPP. Fluid was administered $(10 \mathrm{~mL} / \mathrm{kg} / \mathrm{hr}$ Jonosteril), and the respirator settings for mechanical ventilation were as follows: PEEP of 10 $\mathrm{cm} \mathrm{H}_{2} \mathrm{O}, \mathrm{FiO}_{2}$ of 0.3 , and $\mathrm{I} / \mathrm{E}$ ratio of 1:1.5. Temperature management aimed to achieve brain normothermia. Every 12 hours, the anesthesia depth was reduced until ad- 


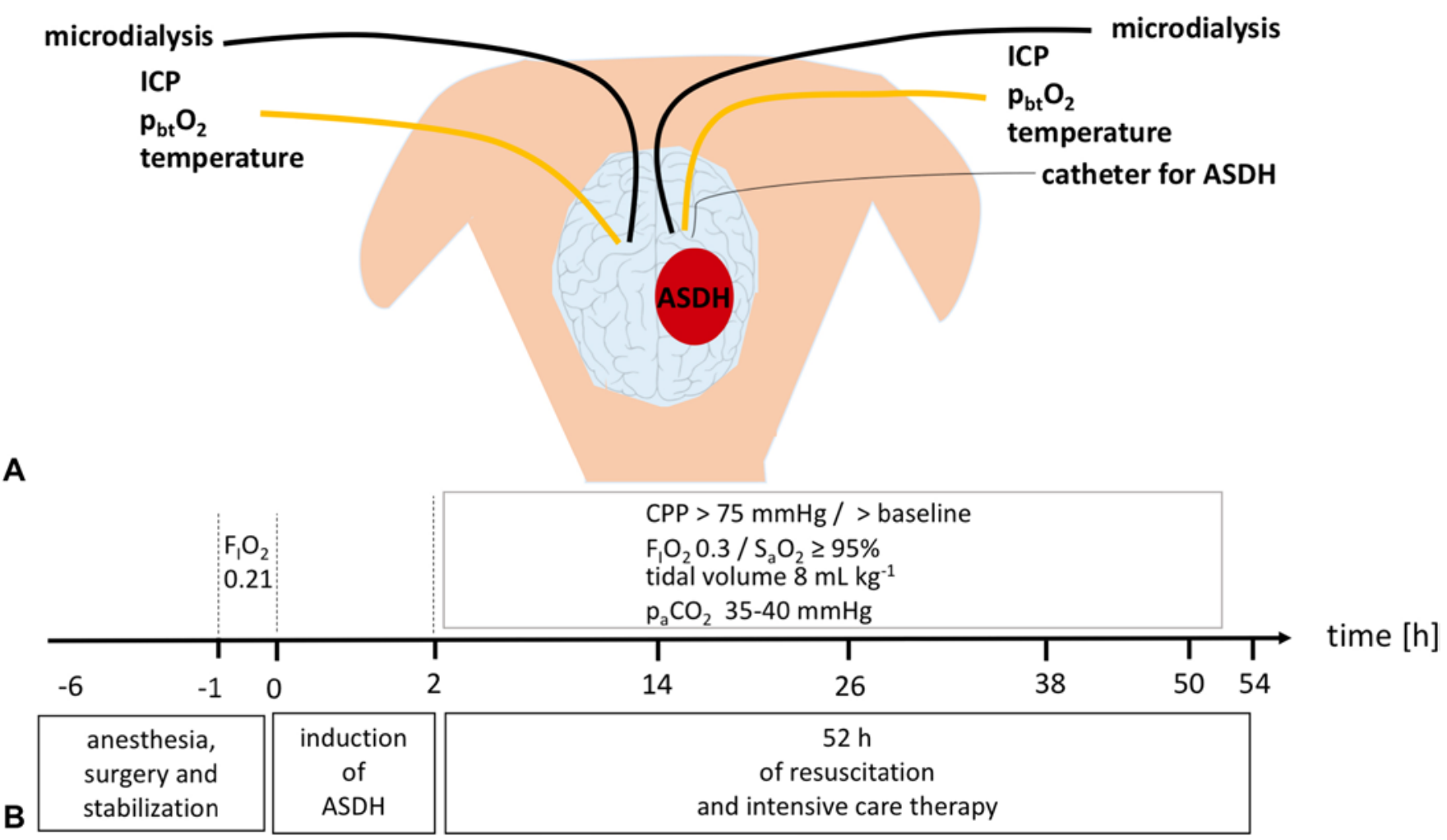

FIG. 1. A: Neurosurgical instrumentation. Via craniotomy above the left and right parietal cortices, microdialysis and parenchymal multimodal probes were inserted on the ipsilateral/left and contralateral/right sides. After exposing the dura on the left side, a small incision was made, and a catheter was inserted $5 \mathrm{~mm}$ into the subdural space for later initiation of the ASDH. The probes were placed under visual control for parenchymal ICP monitoring and $\mathrm{PbtO}_{2}$ and brain temperature measurements. B: Experimental protocol. At least 1 hour before induction of the $\mathrm{ASDH}, \mathrm{FiO}_{2}$ was reduced to 0.21 and $\mathrm{PEEP}$ to $0 \mathrm{~cm} \mathrm{H}_{2} \mathrm{O}$, and the I/E ratio was set to 1:2 for subsequent baseline measurements. After the ASDH-induction phase, the following standard resuscitation protocol was used: CPP was titrated to levels before the induction of the ASDH (if CPP remained below baseline values despite volume resuscitation, norepinephrine was used to restore CPP), while fluid administration and lung-protective mechanical ventilation were used (respirator settings: PEEP $10 \mathrm{~cm} \mathrm{H}_{2} \mathrm{O}, \mathrm{FiO}_{2}$ 0.3, and I/E ratio 1:1.5). $\mathrm{FiO}_{2}$ was stepwise adjusted to maintain an arterial hemoglobin oxygen saturation $\left(\mathrm{SaO}_{2}\right) \geq 95 \%$; temperature management was aimed to achieve normothermia in the brain. Every 12 hours, after reduction of the anesthesia depth, a porcine-adapted MGCS score (adapted from that of Platt et al. ${ }^{35}$ ) was used for neurological assessment. Figure is available in color online only.

equate spontaneous breathing resumed, and neurological function was assessed using a swine-adapted modified Glasgow Coma Scale (MGCS; Table 1). ${ }^{35}$ At the end of the experiments, i.e., after 54 hours of intensive care, after further deepening of anesthesia, the pigs were euthanized via injection of $\mathrm{KCl}$. We strove to make the observation period as long as possible, with termination after 54 hours being imposed by practical limitations, in particular, personnel available for providing ICU care and sample processing.

Intracerebral tissue metabolites were determined using an automated microdialysis system (CMA 600 Microdialysis Analyzer, $\mathrm{CMA}$ /Microdialysis $\mathrm{AB}$ ) equipped for glutamate, lactate, pyruvate, and glucose. Microdialysis catheters (70 microdialysis bolt catheter, M Dialysis AB) were implanted bilaterally after perforation of the dura, and lowered to a depth of $10-15 \mathrm{~mm}$ and perfused (perfusion fluid, CMA/Microdialysis AB) by a microdialysis pump (CMA/102 microdialysis pump, CMA/Microdialysis $\mathrm{AB}$ ). After calibration according to the manufacturer's specifications, microdialysate samples were collected in microvials (MDialysis AB) over 3 hours (i.e., from 1 hour before until 2 hours after each measurement time point) and analyzed immediately after collection. Due to technical failure of the automated analyzing system, there were numerous dropouts and/or implausible values for the glutamate and pyruvate measurements, so that only lactate and glucose data are presented.

Serum blood levels of S100 $\beta$, MAP-2, GFAP, and neuron-specific enolase (NSE) were determined using commercially available porcine specific kits (S100 $\beta$, MAP-2, GFAP, and NSE, all BlueGene BioTech).

As a marker of lipid peroxidation, 8-isoprostane plasma concentrations were measured using a commercially available immunoassay kit (ELISA kit, Cayman Chemicals). Plasma nitrate+nitrite levels, the stable metabolites of nitric oxide (NO), were analyzed with the chemiluminescence method after reduction of nitrate+nitrite to $\mathrm{NO}$ with vanadium chloride (Sievers NOA 280i analyzer, GE Analytical Instruments). Blood samples for these measurements were taken before as well as 2, 14, 26, 38, 50, and 54 hours after induction of the ASDH (see Fig. 1). 


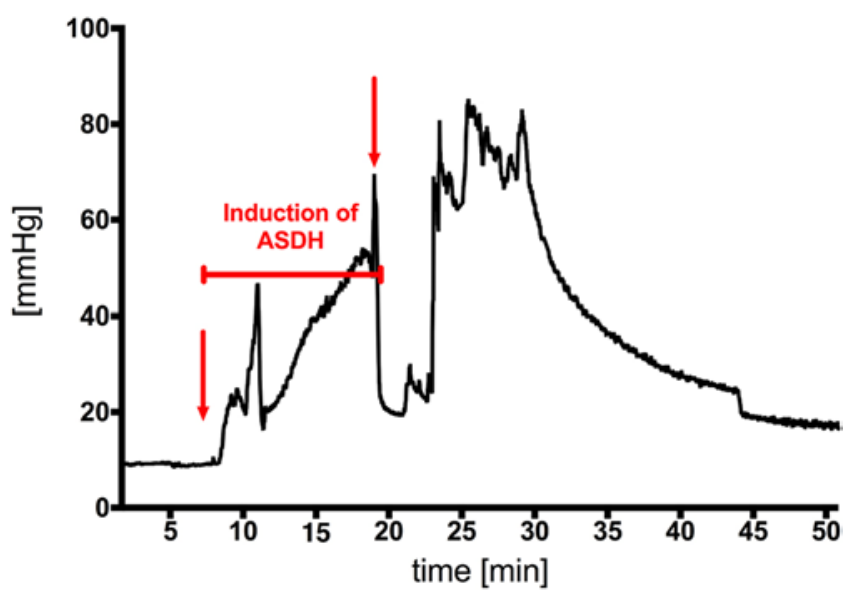

FIG. 2. Graph showing an exemplary time course of the ICP during and immediately after ASDH induction over the first 30 minutes (recording frequency $1 \mathrm{~Hz}$ ). Twenty milliliters of autologous nonheparinized blood was injected using an automated syringe pump via the subdural catheter above the left parietal cortex. Red arrows mark the beginning (left arrow) and the end (right arrow) of the injection time. The temporary drop immediately after termination (right arrow) of the ASDH induction is due to the turning of the 3-way stopcock and the closure of the catheter. Figure is available in color online only.

Homogenized brain tissue specimens from regions adjacent to the surgical instrumentation sites of both sides (brain area next to the ASDH ipsilateral and corresponding right area) were analyzed immediately postmortem for mitochondrial respiratory activity using "high-resolution respirometry" (Oxygraph-2K, Oroboros Instruments). After supplementation of substrates for complexes I and II and $\mathrm{ADP}$, the respiratory capacity in the state of oxidative phosphorylation (coupled state, OXPHOS) was assessed. Maximal respiratory capacity of the electron transfer system (ETS) in the uncoupled state was measured after the addition of 4-(trifluoromethoxy) phenylhydrazone (FCCP). LEAK respiration compensating for proton leakage or slipping was reported as a percentage of ETS capacity. Data are normalized for tissue wet weight.

Immediately postmortem, animals were decapitated, and the brain was removed. The fresh brain was cut sagittally to separate the left and right hemispheres; for protein biochemistry, we sampled an entire slice (5-6 mm thick) from the right hemisphere of the parietal cortex, and for mitochondrial measurements, we took small tissue samples $(300 \mathrm{mg}$ ) of both the ipsi- and contralateral prefrontal cortex. The remaining brain was fixed for 6 days in $4 \%$ formalin. Then, the brain was sectioned in 4-mm sections from frontal to occipital, which were then embedded in paraffin. Standard 3- to 5- $\mu \mathrm{m}$, and, using a modified protocol, $70-\mu \mathrm{m}$, paraffin thick sections were cut and mounted on Superfrost plus (VWR International) slides. General neuropathological evaluation was performed on $\mathrm{H} \& \mathrm{E}$-stained sections.

Immunohistochemical evaluation of the brain slices around the surgical instrumentation sites (brain area next to the ASDH ipsilateral and corresponding right area) was performed for formation of 3-nitrotyrosine, which mirrors the reaction of the superoxide radical with NO to peroxyni-
TABLE 1. MGCS adapted to swine

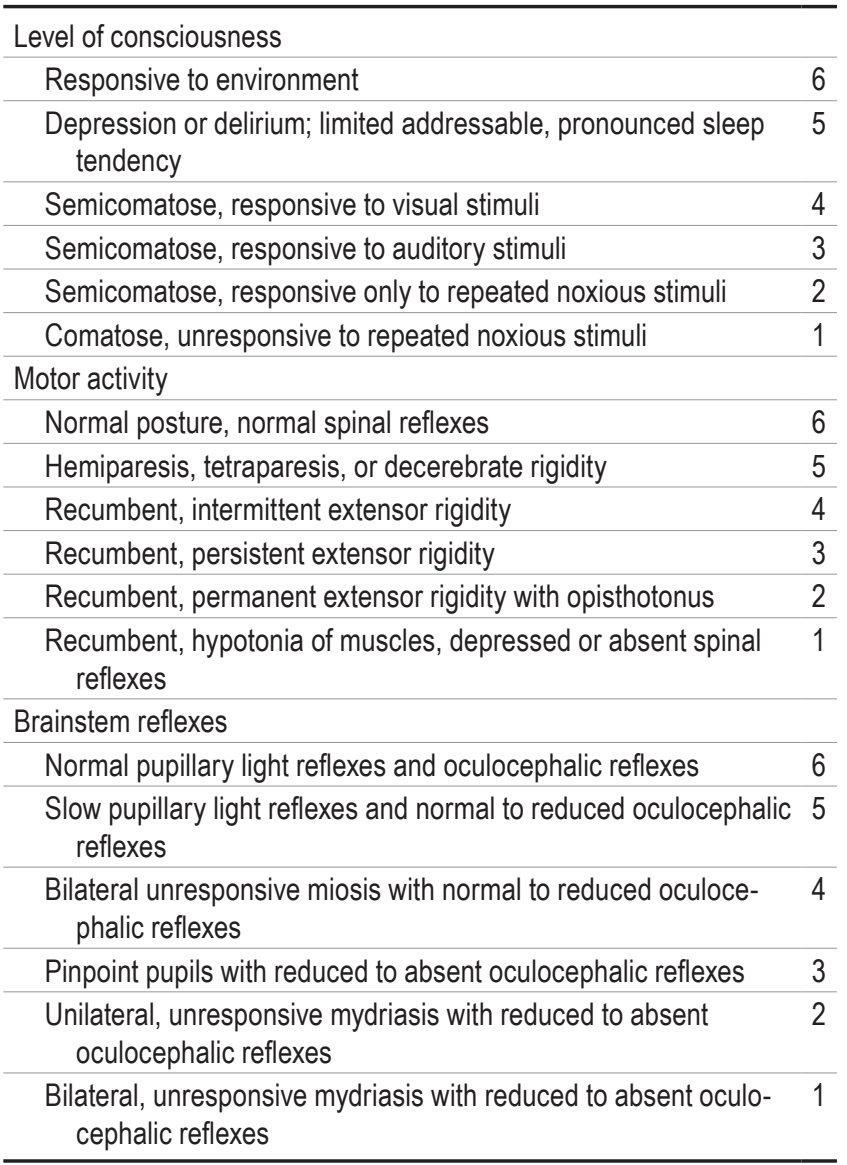

This scale was used for the intermittent neurological assessment. Modified from Platt SR, Radaelli ST, McDonnell JJ: The prognostic value of the modified Glasgow Coma Scale in head trauma in dogs. J Vet Intern Med 15:581-584, 2001, John R. Wiley \& Sons, Inc., (American College of Veterinary Internal Medicine, with permission.

trite; for NADPH oxidase (NOX) expression, the subtype NOX 2 of which is referred to be mainly responsible for reactive oxygen species (ROS) production in brain tissue; ${ }^{26}$ for extravasation of albumin as a marker of blood-brain barrier dysfunction; and for brain tissue, GFAP, a marker of reactive gliosis after brain injury. ${ }^{40}$ The sections were deparaffinized and microwaved for 90 seconds in citrate buffer ( $\mathrm{pH}$ 6.0) for heat-induced antigen retrieval, blocked for 30 minutes in $10 \%$ goat serum, and incubated with the following antibodies: rabbit anti-nitrotyrosine (Merck Millipore), rabbit anti-GFAP (Abcam), rabbit anti-IBA1 (Abcam), mouse anti-NOX2 (gp91phox, Becton Dickinson Bioscience), and rabbit anti-albumin (Abcam). Primary antibody detection was performed using the Dako REAL detection system (anti-mouse, anti-rabbit, Dako) and visualized with red chromogen (DakoREAL) followed by counterstaining with Mayer's hematoxylin (Sigma-Aldrich). Histomorphology was visualized using an Axio Imager.A1 microscope (Zeiss) with a $\times 10$ objective. Two random $800,000-\mu \mathrm{m}^{2}$ regions were quantified for intensity using the AxioVision 4.8 software (Zeiss). Results are presented as mean densitometric sum red. 


\section{Results}

Table 2 summarizes systemic hemodynamics, acidbase state, gas exchange, lung mechanics, and metabolism. The pigs' temperatures dropped during the surgery and were restored to normal levels after 14 hours of resuscitation. Except for a statistically significant, but clinically irrelevant, decrease over time of glycemia, lactatemia levels, and the right carotid artery flow, all parameters remained stable throughout the experiment. In all animals, macroscopic evaluation at necropsy showed a solid subdural clot of about $2 \mathrm{~cm}$ in diameter on the left cortical surface (Fig. 3). In addition, in all animals hemorrhage had broken through the subarachnoid into the intracerebral space, with bleeding reaching the lateral ventricles in 3 animals.

Figure 2 demonstrates that ICP sharply rose on ASDH induction and remained increased in both hemispheres until the end of the experiment. Table 2 also summarizes the results of the multimodal brain monitoring. At 36 hours of intensive care, i.e., 38 hours after ASDH induction, the ICP in the left hemisphere was significantly higher than that in the right hemisphere $(p=0.024)$. Despite the increased ICP, fluid resuscitation and continuous intravenous norepinephrine needed by all animals (median/ IQR infusion rate median $0.10 \mu \mathrm{g} / \mathrm{kg} / \mathrm{min}$ [IQR $0.04-0.39$ $\mu \mathrm{g} / \mathrm{kg} / \mathrm{min}]$ ) allowed the CPP to be maintained at baseline levels. Strikingly, $\mathrm{PbtO}_{2}$ values significantly differed between the two hemispheres at baseline $(\mathrm{p}=0.048)$, but this difference disappeared throughout the experiment; overall, $\mathrm{PbtO}_{2}$ was not significantly affected over time. Despite the macroscopically visible damage, the well-maintained $\mathrm{CPP}$ and unchanged $\mathrm{PbtO}_{2}$ were associated with a stable MGCS score throughout the entire experiment.

Figure 4 shows the brain microdialysis glucose and lactate concentrations. At baseline the median lactate levels were $2.2 \mathrm{mmol} / \mathrm{L}$ (IQR $1.0-4.0 \mathrm{mmol} / \mathrm{L}$ ) in the ipsilateral/ left hemisphere and $1.7 \mathrm{mmol} / \mathrm{L}$ (IQR $0.2-2.6 \mathrm{mmol} / \mathrm{L}$ ) in the contralateral/right hemisphere. After ASDH induction, left-sided lactate levels increased up to a median of $2.9 \mathrm{mmol} / \mathrm{L}$ (IQR $1.4-4.4 \mathrm{mmol} / \mathrm{L}$ ), however, with pronounced interindividual variation, but remained stable in the right hemisphere. Tissue glucose levels at baseline were $0.4 \mathrm{mmol} / \mathrm{L}$ (IQR $0.3-1.0 \mathrm{mmol} / \mathrm{L}$ ) in the ipsilateral and $0.4 \mathrm{mmol} / \mathrm{L}(0.1-0.6 \mathrm{mmol} / \mathrm{L})$ in the contralateral hemispheres. The ipsilateral glucose concentrations significantly decreased to values of $0.01 \mathrm{mmol} / \mathrm{L}$ (IQR $0.01-$ $0.3 \mathrm{mmol} / \mathrm{L}$ ) over time, whereas they remained unchanged in the right hemisphere. Again, there were considerable interindividual variations.

The systemic markers of NO and ROS formation as well as brain injury are summarized in Table 2. Except for the S100 $\beta$, which significantly decreased over time, none of these parameters (8-isoprostane, nitrite+nitrate, GFAP, NSE, or MAP-2) were significantly affected. Figure 4 shows that mitochondrial respiratory activity of immediate postmortem brain specimens taken from regions adjacent to the surgical instrumentation did not differ between the two hemispheres.

Figure 5 shows typical examples of the histological findings: all swine showed periventricular lymphocyte infiltration, the degree of which was moderate and differed among individual animals. Three animals also showed minor granulocyte infiltration, possibly documenting secondary infection associated with the surgical instrumentation. There were no significant histological signs of tissue hypoxia, although dark eosinophilic neurons were found in the injured hemisphere near the ASDH site.

Figure 6 shows typical examples as well as the quantitative analysis of the immunohistochemical evaluation. ASDH induction was associated with marked 3-nitrotyrosine formation, extravascular albumin accumulation, and NOX2 expression, which coincided with a significantly higher GFAP expression in the cortex next to the ASDH compared with the corresponding region in the contralateral hemisphere. Moreover, analysis of the dorsomedial prefrontal cortex with a distant location to the ASDH site revealed GFAP-positive astrogliosis and more complex morphologies of IBA-1 immunoreactive cells, indicating microglial activation on the left hemisphere with ASDH, whereas these changes were not present in the contralateral dorsomedial prefrontal cortex.

\section{Discussion}

The purpose of this study was to describe a clinically relevant, long-term, resuscitated porcine model of ASDHinduced acute brain injury. The main results were as follows: 1) ASDH caused nitrosative and oxidative stress, while the neurosurgical instrumentation alone had no effect. 2) Local brain injury was not reflected by systemic markers of oxidative or nitrosative stress or markers of brain injury. 3) Despite the morphological damage, maintenance of CPP and $\mathrm{PbtO}_{2}$ prevented severe impairment of tissue energy metabolism and, hence, serious neurological dysfunction.

We chose ASDH as model of acute brain injury because of its reproducibility and clinical relevance, ${ }^{18,23,44}$ which resulted in different manifestations of brain injury beyond the subdural hemorrhage, possibly due to secondary bleeding and/or pressure-related rupture of the arachnoid and pia maters. Since the results of all investigated parameters were similar, we suspect that the bleeding in the lateral ventricles mirrored different manifestations due to anatomical variations. Porcine models of ASDH using multimodal brain monitoring have been described for up to 12 hours only using injected blood volumes of 2-9 mL. ${ }^{30,41}$ We injected $20 \mathrm{~mL}$ of blood into the subdural space, corresponding to $12.5 \%$ of the brain volume, because previous studies on porcine ASDH had used comparable ratios $(10 \%-15 \%)$ of the volume of subdural blood injected and brain volume..$^{30,38,41}$ In contrast to the aforementioned studies, ${ }^{30,41}$ we used human-sized animals with a median body weight of $65 \mathrm{~kg}$ and, consequently, weight-adapted the amount of blood injected into the subdural space. The ICP increased in both sides with higher ipsilateral values throughout the experiment, with the contralateral ICP increase most likely being mass effect due to intracranial pressure spreading. The present model focused on the effects of an ASDH-induced brain injury in human-sized adult swine together with the integration of guideline-based intensive care therapy. Hence, it is likely that the long-term anesthesia and the surgical instrumentation also affected our findings, in particular with respect 


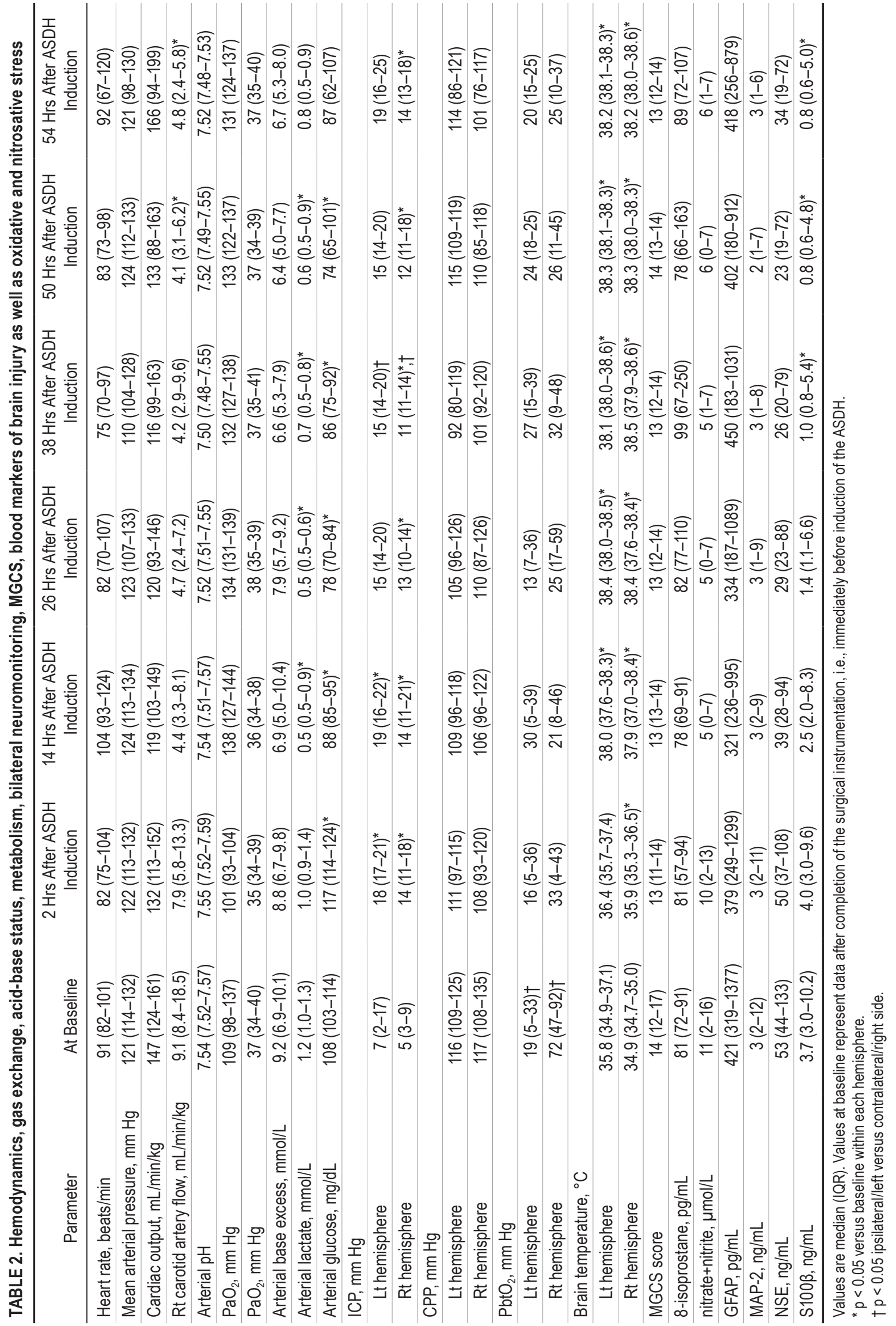



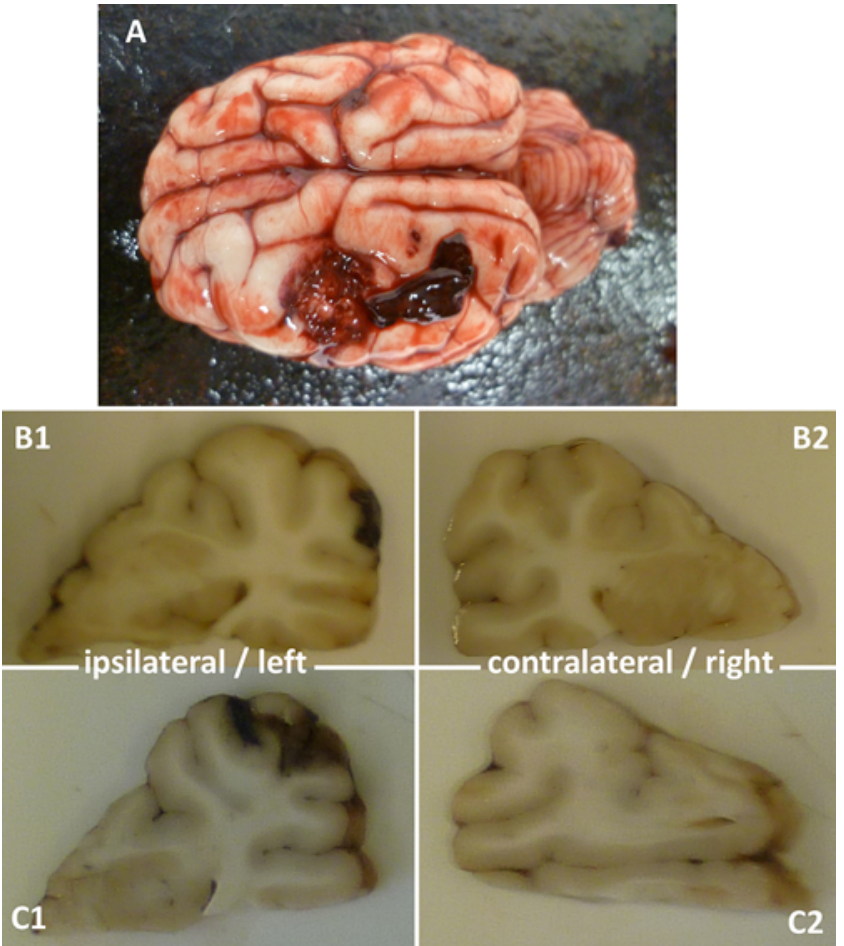

FIG. 3. Photograph of a porcine brain immediately after termination of the experiment $(\mathbf{A})$, showing a solid clot above the left hemisphere that has just released from its still-visible original position. Macroscopic visible damage of the formalin-fixed brain slices on the ipsilateral/left hemisphere (B1 and $\mathbf{C} 1$ ) is contrasted with its contralateral/right hemisphere (B2 and C2). Figure is available in color online only.

to the systemic biomarkers. However, investigation of pathophysiological challenges and/or possible therapeutic interventions during acute hemorrhage or trauma-induced brain injury using current resuscitation standards would also require long-term anesthesia and surgical instrumentation, and, therefore, control animals without anesthesia, surgery, and/or baseline injury would have provided data from completely different conditions. We performed identical, bilateral neurosurgical instrumentation procedures on the left (ipsilateral) and on the right (contralateral) hemispheres so that the "sham-instrumented" hemisphere could be used as a control for the ASDH hemisphere. Due to the significant macroscopic (Fig. 3) and immunohistochemical (Fig. 6) differences (in particular with respect to 3-nitrotyrosine formation, albumin extravasation, and NOX2 expression) between both sides, we strongly suggest that the ipsilateral findings were related to the ASDH rather than the neurosurgery. Moreover, this approach also allowed for complying with the $3 \mathrm{R}$ principle to reduce the number of animals. ${ }^{25}$

Experiments lasted for 54 hours under intensive care conditions, including neurological assessment using an adapted MGCS. ${ }^{35}$ To our knowledge, no large-animal model of ASDH has yet been described that includes multimodal brain monitoring and full-scale intensive care over a resuscitation period of 54 hours. A study on ASDH in 3-week-old piglets described neurological deficits at days 3 and $7,{ }^{38}$ but these animals did not receive intensive care.
Other porcine studies on $\mathrm{ASDH}^{30,41}$ did not describe any detailed neurological assessment. Clearly, MGCS scores in our study were lower than normal values. However, baseline assessment was taken after the end of the neurosurgical instrumentation, and, therefore, the depth of anesthesia was only reduced to levels allowing for spontaneous breathing rather than complete awake status. Nevertheless, neither ASDH induction nor prolonged anesthesia had any further effect on the neurological function.

In contrast to earlier large-animal studies of ASDH-induced and/or traumatic brain injury, ${ }^{1,12,28,38,41}$ we measured ICP bilaterally. Since systemic hemodynamics remained stable due to fluid resuscitation and norepinephrine infusion, CPP was well maintained throughout the experiment, which was ultimately associated with an unaffected MGCS score. These observations are in line with a shortterm (6 hours) study of acute traumatic brain injury in piglets, which showed attenuated metabolic disturbances and brain injury when a CPP of $70 \mathrm{~mm} \mathrm{Hg}$ rather than $40 \mathrm{~mm}$ Hg was targeted. ${ }^{12}$

Monitoring of $\mathrm{PbtO}_{2}$ to detect cerebral ischemia has been advocated for the management of patients after severe head injury. ${ }^{42}$ In that study, the duration of periods with $\mathrm{PbtO}_{2} \leq 15 \mathrm{~mm} \mathrm{Hg}$ were directly related to poor neurological outcome and mortality. ${ }^{42}$ In our study, $\mathrm{PbtO}_{2}$ values remained unchanged over time and did not show statistically significant differences between the two hemispheres. However, data recorded showed large variations, probe malfunctions with implausible values and, consequently, various data could not be used. Moreover, individual values were below the above-mentioned threshold, indicating regional cerebral hypoxia and/or ischemia. At first glance, this finding is in contrast to the unaffected MGCS scores. However, comparably broad variations of $\mathrm{PbtO}_{2}$ values with standard deviations of up to $50 \%$ of the recorded mean values were observed in a pig study on brain death due to a gradual increase in ICP resulting from inflation of an epidural balloon. ${ }^{36} \mathrm{~A}$ more recent study characterizing a multiparameter brain probe in juvenile pigs (maximum duration 5-6 hours) undergoing various physiological challenges (hypoxia, hypercapnia, norepinephrine infusion) with or without cortical impact also reported individual $\mathrm{PbtO}_{2}<15 \mathrm{~mm} \mathrm{Hg}$ with neurosurgical instrumentation alone as well as standard deviations of the recorded mean $\mathrm{PbtO}_{2}$ levels of up to $50 \%$. In that study, approximately $20 \%$ of the recorded $\mathrm{PbtO}_{2}$ values had to be discarded completely. ${ }^{27}$

Microdialysis lactate concentrations in the ASDH-challenged hemisphere increased up to $2.9 \mathrm{mmol} / \mathrm{L}$ without significant differences from baseline or between the hemispheres. Microdialysis glucose levels significantly decreased in the ipsilateral, hematoma-affected hemisphere to values $<0.1 \mathrm{mmol} / \mathrm{L}$, whereas the contralateral hemisphere did not show any significant effect. Our findings are in line with a porcine study of controlled cortical impact reporting microdialysis lactate values up to $2.16 \mathrm{mmol} / \mathrm{L}$ and a drop of glucose values to $0.1 \mathrm{mmol} / \mathrm{L} .{ }^{1}$ Comparable microdialysis data were shown in a brain-death study in pigs with lactate values of up to $3.0 \mathrm{mmol} / \mathrm{L}$ and glucose levels of $0.1 \mathrm{mmol} / \mathrm{L} .{ }^{27}$ The nonsignificant decrease of microdialysis lactate during the experiment was most likely 

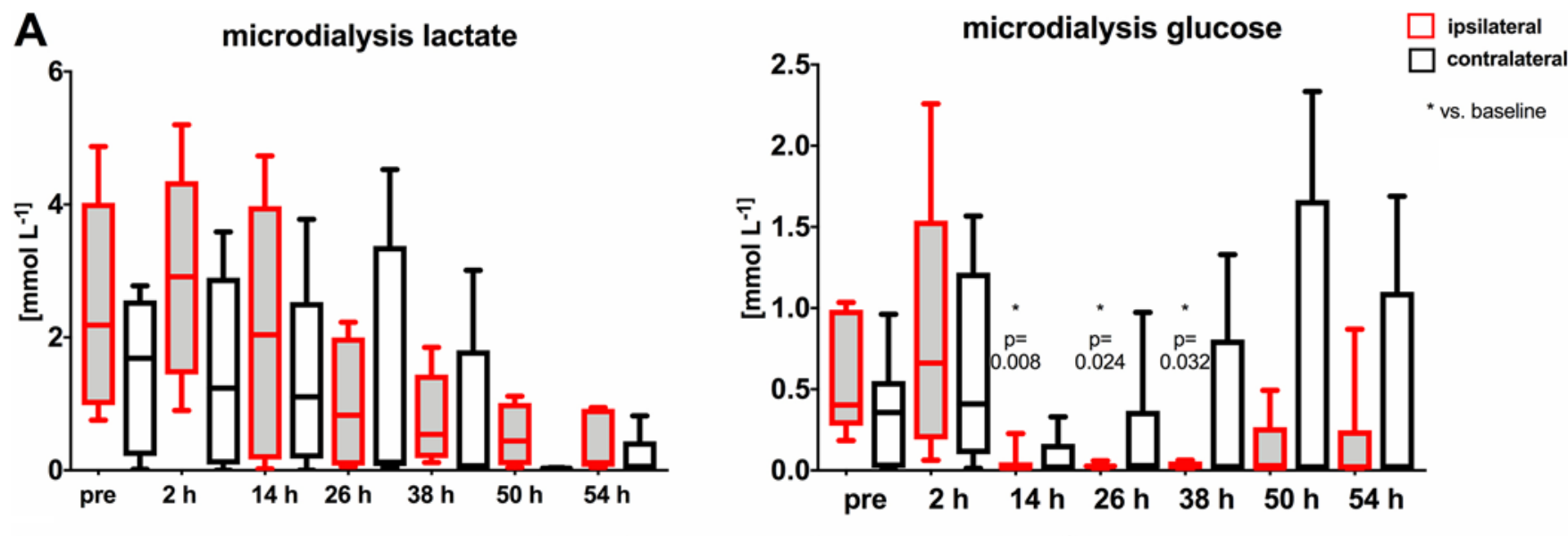

B

mitochondrial respiratory capacity of brain

OXPHOS

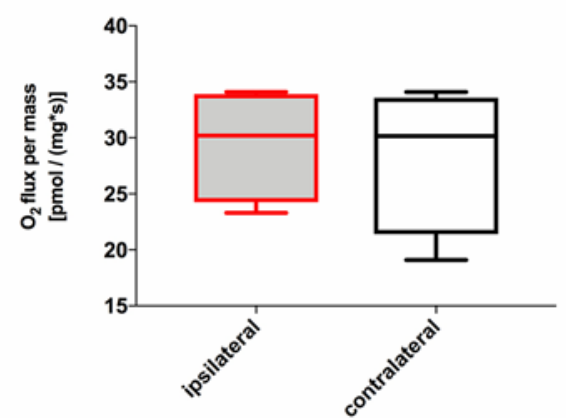

ETS

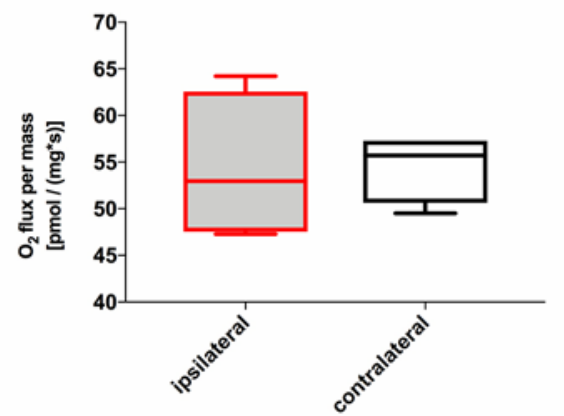

LEAK/ETS

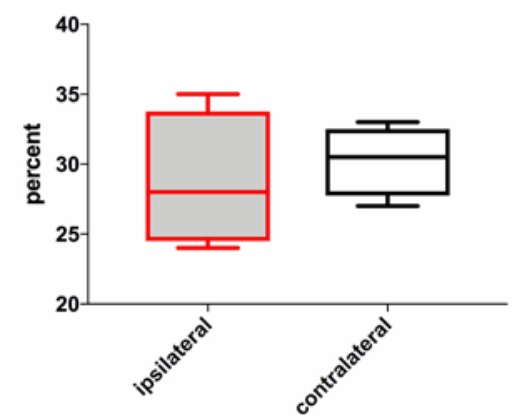

FIG. 4. A: Microdialysis lactate and glucose levels are presented as boxplots (median, IQR, maximum/minimum) over time at baseline (pre) and after (2-54 hours) the induction of the ASDH of the ipsilateral (left, with the ASDH) and the contralateral (right) hemisphere. *Significantly different from baseline in the ipsilateral glucose concentrations. B: Mitochondrial respiratory capacity of brain samples. Mitochondrial function was analyzed using high-resolution respirometry (Oroboros Oxygraph-2K, Oroboros Instruments) in homogenized tissue specimens collected immediately postmortem from brain regions directly adjacent to the surgical instrumentation site. ETS = maximal respiratory capacity of the electron transfer system in the uncoupled state; LEAK = respiration compensating for proton leakage or slipping and is reported as percentage of ETS; OXPHOS = mitochondrial respiratory capacity in the state of oxidative phosphorylation (coupled state). Data are normalized for tissue wet weight and represent 4 animals. Figure is available in color online only.

observed because of the CPP maintenance and the volume substitution during the intensive care therapy. Due to the missing data of microdialysis glutamate and pyruvate in the present study, the validity of the microdialysis measurements may be regarded as questionable. However, previous studies on porcine ASDH by Alessandri et al. ${ }^{1}$ and Purins et al. ${ }^{36}$ showed similarly huge variations in microdialysis glutamate and pyruvate, the overall data being comparable to our findings. Clearly, the metabolic disturbances were only present in the hematoma-carrying hemisphere, and less pronounced than in these authors' studies. Again, this observation is most likely due to the strict adherence to a brain-targeted resuscitation protocol in our study.

The S100 $\beta$ plasma concentrations significantly decreased throughout the experiment. Increased $S 100 \beta$ plasma levels have been reported in porcine traumatic brain injury. ${ }^{13}$ However, a study in piglets undergoing cortical impact did not show any increase of $S 100 \beta,{ }^{6}$ and another study on hypothermic cardiac arrest in juvenile pigs only described an initial, transitory rise of $S 100 \beta$, which then decreased even below baseline values in the further exper- iment. ${ }^{37}$ In the present study, high levels of S100 $\beta$ were already present at baseline (median $3.7 \mathrm{ng} / \mathrm{mL}$ [IQR 3.0-10.2 $\mathrm{ng} / \mathrm{mL}]$ ), and decreased below baseline levels until the end of the experiments. Different time courses of S100 $\beta$ might be related to various potential sources of S100 $\beta .{ }^{19}$ Hence, our study confirms the limits of this parameter to mirror severity of brain injury. ${ }^{6,37}$ The interpretation of bloodbrain biomarkers in general is critically discussed, ${ }^{6,13}$ and findings of the present study differ from those of previous studies due to the use of guideline-directed intensive care.

GFAP is described as a marker of the severity of head injury. ${ }^{24,32}$ In the present study, GFAP serum levels did not change significantly, whereas immunohistochemical analysis showed significantly higher GFAP expression in the ipsilateral, hematoma-carrying hemisphere. Increased serum GFAP levels have been reported after cortical impact in swine; however, these animals underwent brain injury together with hemorrhagic shock, i.e., markedly reduced CPP over 2 hours. ${ }^{22}$ Nevertheless, our immunohistochemical findings of markedly higher GFAP expression are in line with those in studies demonstrating hyperintensity of 

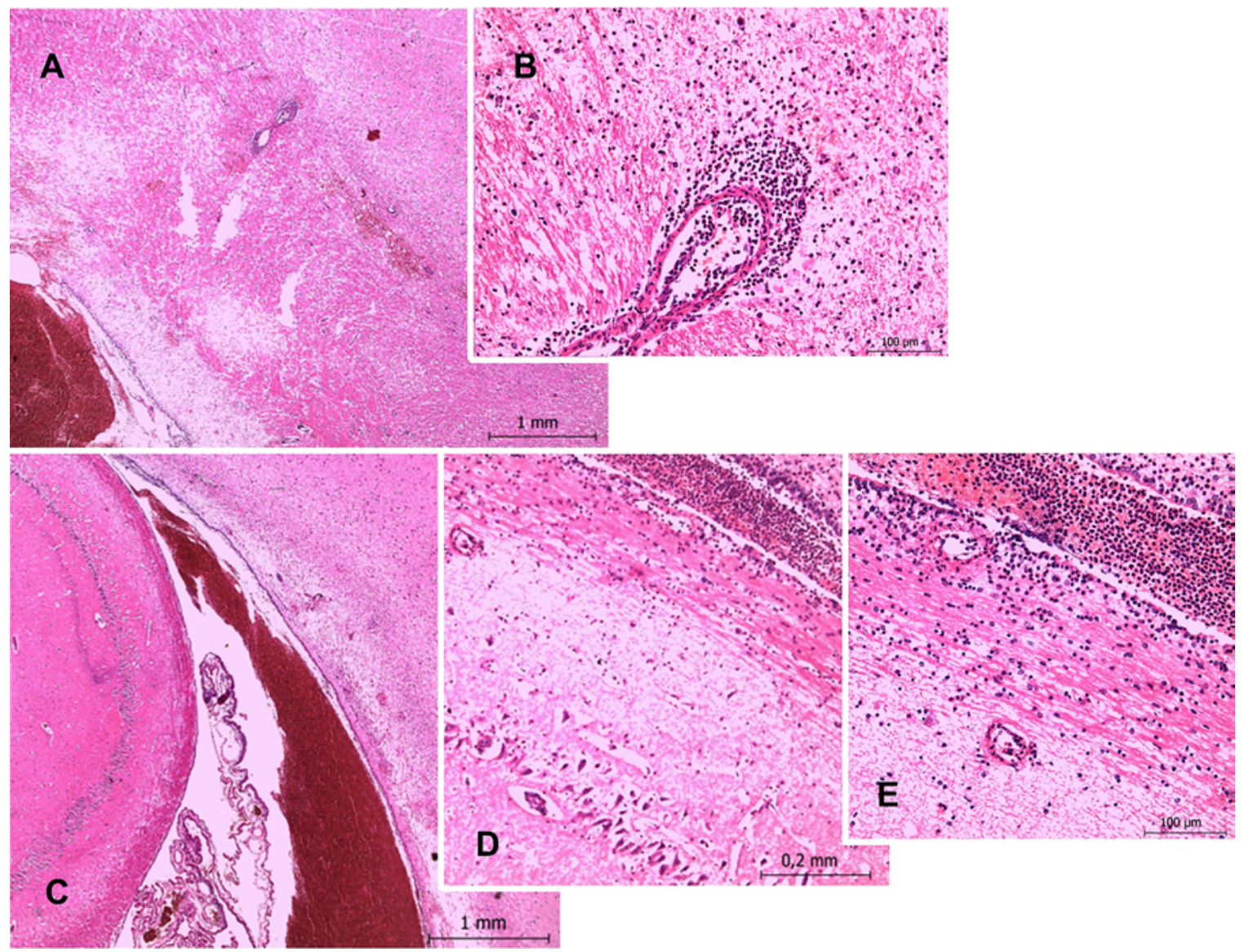

FIG. 5. Histological findings of the brain. Exemplary $\mathrm{H}$ \& E-stained sections. All swine showed periventricular lymphocyte infiltration, the degree of which was moderate and differed between individual animals. In addition, 3 animals also showed minor granulocyte infiltration, most likely documenting secondary infection associated with the surgical instrumentation. There were no significant histological signs of tissue hypoxia, although dark eosinophilic neurons were found in the injured hemisphere near the ASDH site. A and B: Typical perivasal mixed cell (granulocyte and lymphocyte) inflammation. C-E: Reactive inflammatory cells (lymphocytes and granulocytes), which migrate from the ventricle into the brain parenchyma. Bar = $1 \mathrm{~mm}(\mathrm{~A}$ and $\mathrm{C}), 0.2 \mu \mathrm{m}(\mathrm{D})$, and $100 \mu \mathrm{m}(\mathrm{B}$ and $\mathrm{E})$. Figure is available in color online only.

GFAP as an indicator of reactive gliosis after brain injury in rats ${ }^{40}$ and swine. ${ }^{17}$ Moreover, GFAP-positive astrogliosis was accompanied by activation of IBA-1-positive microglial cells, which was not only seen next the ASDH site but also at distant locations.

Plasma NSE remained unaffected in our study. A doubling of NSE serum levels has been reported in piglets on day 1 after cortical impact, regardless of the degree of histological damage. ${ }^{6}$ However, animals in that study did not receive intensive care.

Plasma levels of MAP-2 are suggested as a parameter for ischemic brain injury in humans, ${ }^{34}$ and, in fact, increased MAP-2 levels were found in the CSF in patients with severe traumatic brain injury. ${ }^{33}$ Rats $^{8}$ and swine ${ }^{29}$ showed decreased immunohistochemical MAP-2 expressions from injured brain regions, while increased plasma MAP-2 levels could be detected shortly after ischemia in the rat. ${ }^{34} \mathrm{In}$ contrast, MAP-2 levels did not show any significant changes over time in our present study. This discrepancy is most likely due to unaffected $\mathrm{CPP}$ and $\mathrm{PbtO}_{2}$ values resulting from strict adherence to therapeutic guidelines.
Primary and secondary brain injury are associated with impaired bioenergetics and mitochondrial dysfunction and/or damage. ${ }^{16}$ Significant alterations in cerebral mitochondrial bioenergetics were described in juvenile swine with traumatic brain injury, surprisingly most pronounced in the contralateral, unaffected hemisphere. ${ }^{20}$ In contrast, in our study, mitochondrial respiration did not differ between both hemispheres. However, we investigated adult animals with mature brains, and, again, strictly adhered to brain-targeted intensive care guidelines.

Brain tissue nitrotyrosine expression, a marker of peroxynitrite formation ${ }^{4}$ has been reported in traumatic brain injury in mice ${ }^{14}$ and rats. ${ }^{15}$ In patients with severe brain injury, nitrotyrosine levels in the CSF were indicative of poor neurological outcome. ${ }^{7}$ In the present study, nitrotyrosine and NOX2 as indicators of increased oxidative and nitrosative stress were significantly increased in the ipsilateral, blood-injected hemisphere, when compared with the contralateral hemisphere, whereas systemic markers of $\mathrm{NO}$ and ROS formation, 8-isoprostane and nitrite+nitrate plasma levels, remained unchanged. The ipsilateral nitro- 
A
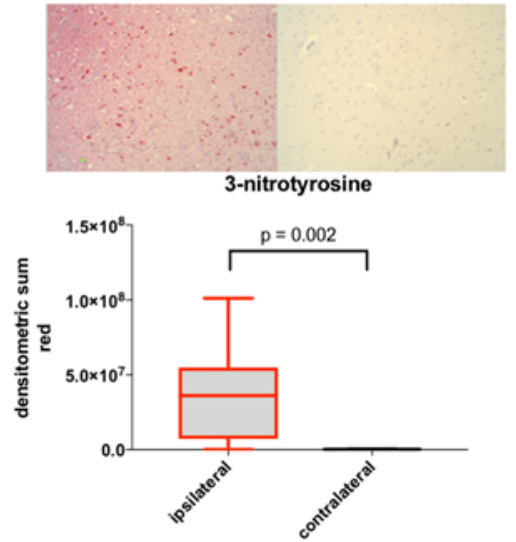

C
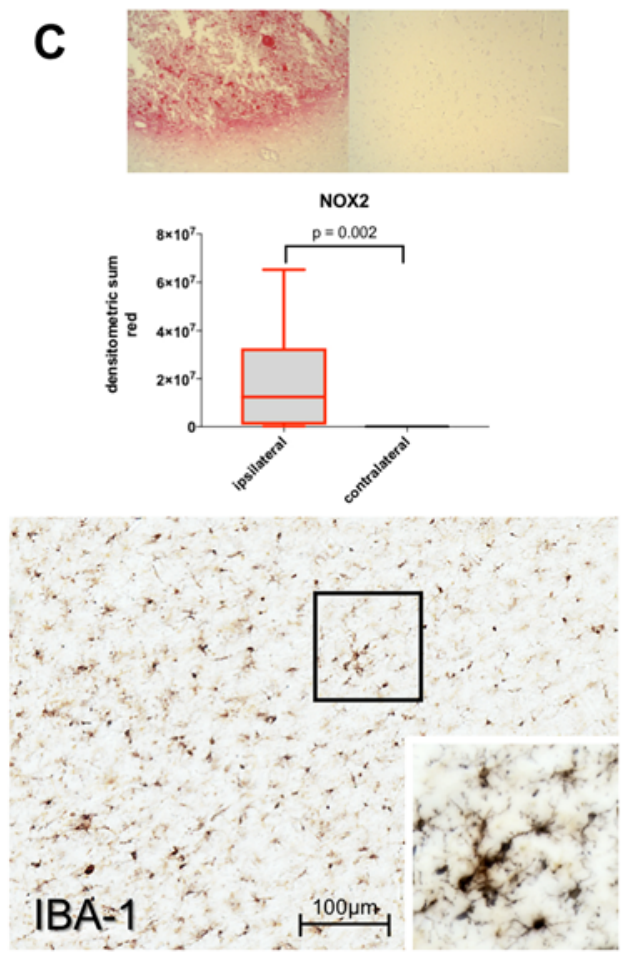

E

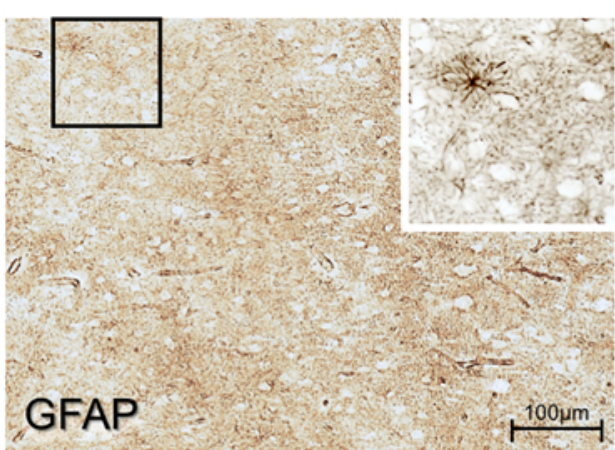

G

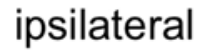

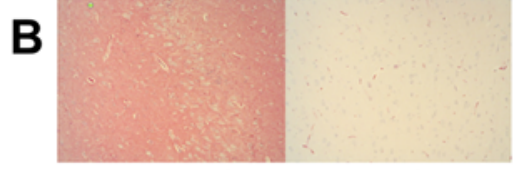

extravascular albumin

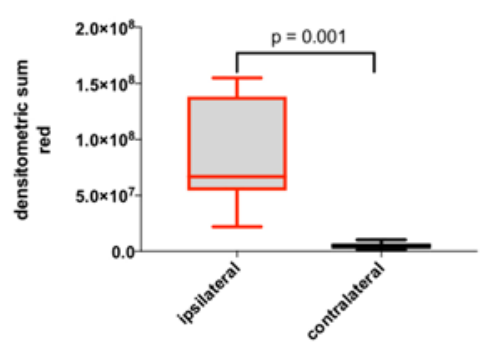

D
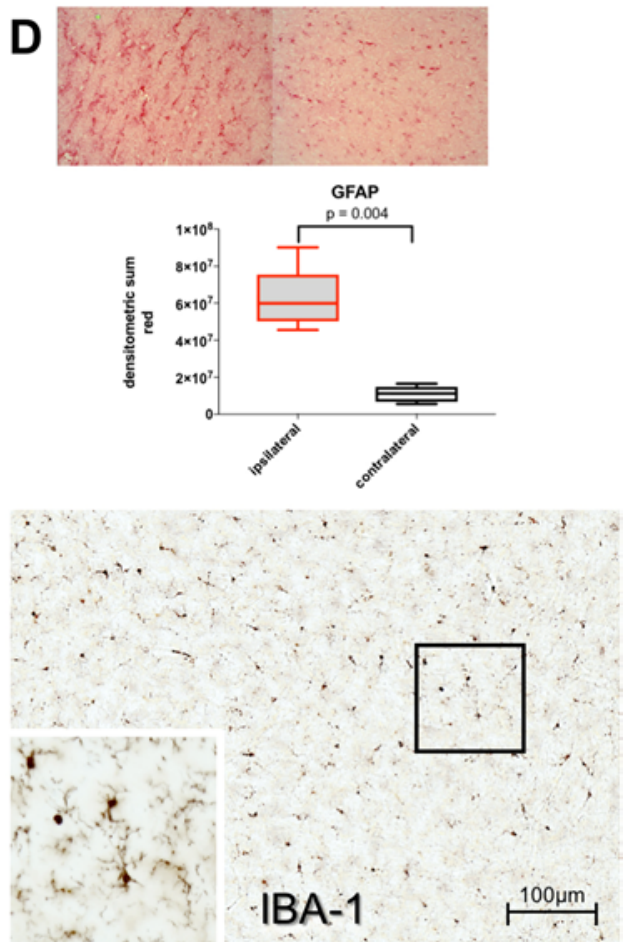

$\mathbf{F}$

FIG. 6. Immunohistochemical analysis of brain specimens and changes in IBA-1 and GFAP immunoreactive cells distant from the ASDH site. A-D: Immunohistochemical analysis of the brain specimens. Examples and quantitative results of the densitometric analysis of animals and representative histological images (original magnification $\times 10$ objective) of the brain immunohistochemistry for 3 -nitrotyrosine $(A ; n=7$ ipsilateral, $n=7$ contralateral), extravascular albumin (B; $n=7$ ipsilateral, $n=7$ contralateral), NOX2 (C; $n=6$ ipsilateral, $n=6$ contralateral), and GFAP (D; $n=6$ ipsilateral, $n=6$ contralateral). Distinct hemisphere cross-sections were dissected, fixed in formalin, dehydrated, and embedded in paraffin. FIG. 6. (continued) $\rightarrow$ 
FIG. 6. Slices of 3-mm thickness were cut and mounted on slides. Primary antibody detection was performed by an alkaline phosphatase-conjugated secondary antibody and visualized with an alkaline phosphatase substrate red chromogen followed by counterstaining with hematoxylin. Two representative $800,000-\mathrm{mm}^{2}$ sections per slide were graded. Quantification of the intensity of the red chromogen was performed. Results are presented as median densitometric sum red. Boxplots display median, IQR, and range. E-H: Changes in IBA-1 and GFAP immunoreactive cells distant from the ASDH site. The dorsomedial prefrontal cortex displays IBA-1 immunoreactive activated microglial cells and GFAP-positive astrogliosis on the ipsilateral hemisphere ( $E$ and $G$ ) subjected to $A S D H$ as seen in 70 - $u$ m-thick brain sections. In the dorsomedial prefrontal cortex of the contralateral hemisphere ( $F$ and $\mathrm{H}$ ), resting IBA-1-positive microglia are found, and astrogliosis is mild. Bar $=100 \mu \mathrm{m}(\mathrm{E}-\mathrm{H})$. Figure is available in color online only.

tyrosine formation is in line with those in these previous studies, ${ }^{14,15}$ while the unchanged serum markers indicate that maintenance of $\mathrm{CPP}$ and $\mathrm{PbtO}_{2}$ might prevent signs of systemic oxidative and nitrosative stress.

\section{Conclusions}

We describe a clinically relevant, long-term, resuscitated porcine model of ASDH-induced acute brain injury. ASDH was associated with pronounced morphological damage, which was only related to the hematoma, whereas the neurosurgical instrumentation alone had no effect. Local brain injury was not reflected by systemic markers, most likely as a result of maintenance of $\mathrm{CPP}$ and $\mathrm{PbtO}_{2}$, which in turn prevented impairment of tissue energy metabolism and, subsequently, major neurological dysfunction. This model is suitable for studying pathophysiological challenges and/or therapeutic interventions during acute hemorrhage- and/or trauma-induced brain injury, under current resuscitation standards.

\section{Acknowledgments}

The study was supported by a research grant from the Deutsche Forschungsgemeinschaft, Bonn, Germany (SFB 1149); Gerok (M.W.); promotion by faculty of medicine (Baustein-Förderung) (B.L.N.); and Hertha Nathorff-Programm (C.H.).

\section{References}

1. Alessandri B, Heimann A, Filippi R, Kopacz L, Kempski O: Moderate controlled cortical contusion in pigs: effects on multi-parametric neuromonitoring and clinical relevance. $\mathbf{J}$ Neurotrauma 20:1293-1305, 2003

2. Anderson RW, Brown CJ, Blumbergs PC, McLean AJ, Jones NR: Impact mechanics and axonal injury in a sheep model. J Neurotrauma 20:961-974, 2003

3. Armstead WM, Riley J, Vavilala MS: Sex and age differences in epinephrine mechanisms and outcomes after brain injury. J Neurotrauma 34:1666-1675, 2017

4. Beckman JS, Koppenol WH: Nitric oxide, superoxide, and peroxynitrite: the good, the bad, and ugly. Am J Physiol 271:C1424-C1437, 1996

5. Carney N, Totten AM, O'Reilly C, Ullman JS, Hawryluk GW, Bell MJ, et al: Guidelines for the Management of Severe Traumatic Brain Injury, Fourth Edition. Neurosurgery 80:6-15, 2017

6. Costine BA, Quebeda-Clerkin PB, Dodge CP, Harris BT, Hillier SC, Duhaime AC: Neuron-specific enolase, but not S100B or myelin basic protein, increases in peripheral blood corresponding to lesion volume after cortical impact in piglets. J Neurotrauma 29:2689-2695, 2012

7. Darwish RS, Amiridze N, Aarabi B: Nitrotyrosine as an oxidative stress marker: evidence for involvement in neurologic outcome in human traumatic brain injury. J Trauma 63:439-442, 2007

8. Dawson DA, Hallenbeck JM: Acute focal ischemia-induced alterations in MAP2 immunostaining: description of temporal changes and utilization as a marker for volumetric assessment of acute brain injury. J Cereb Blood Flow Metab 16:170-174, 1996

9. DeWitt DS, Hawkins BE, Dixon CE, Kochanek PM, Armstead W, Bass CR, et al: Pre-clinical testing of therapies for traumatic brain injury. J Neurotrauma 35:2737-2754, 2018

10. Duhaime AC: Large animal models of traumatic injury to the immature brain. Dev Neurosci 28:380-387, 2006

11. Durham SR, Duhaime AC: Basic science; maturation-dependent response of the immature brain to experimental subdural hematoma. J Neurotrauma 24:5-14, 2007

12. Friess SH, Smith C, Kilbaugh TJ, Frangos SG, Ralston J, Helfaer MA, et al: Early cerebral perfusion pressure augmentation with phenylephrine after traumatic brain injury may be neuroprotective in a pediatric swine model. Crit Care Med 40:2400-2406, 2012

13. Gyorgy A, Ling G, Wingo D, Walker J, Tong L, Parks S, et al: Time-dependent changes in serum biomarker levels after blast traumatic brain injury. J Neurotrauma 28:1121-1126, 2011

14. Hall ED, Detloff MR, Johnson K, Kupina NC: Peroxynitritemediated protein nitration and lipid peroxidation in a mouse model of traumatic brain injury. J Neurotrauma 21:9-20, 2004

15. Henderson M, Rice B, Sebastian A, Sullivan PG, King C, Robinson RA, et al: Neuroproteomic study of nitrated proteins in moderate traumatic brain injured rats treated with gamma glutamyl cysteine ethyl ester administration post injury: Insight into the role of glutathione elevation in nitrosative stress. Proteomics Clin Appl 10:1218-1224, 2016

16. Hiebert JB, Shen Q, Thimmesch AR, Pierce JD: Traumatic brain injury and mitochondrial dysfunction. Am J Med Sci 350:132-138, 2015

17. Johnson VE, Weber MT, Xiao R, Cullen DK, Meaney DF, Stewart W, et al: Mechanical disruption of the blood-brain barrier following experimental concussion. Acta Neuropathol 135:711-726, 2018

18. Karibe H, Hayashi T, Hirano T, Kameyama M, Nakagawa A, Tominaga T: Surgical management of traumatic acute subdural hematoma in adults: a review. Neurol Med Chir (Tokyo) 54:887-894, 2014

19. Kawata K, Liu CY, Merkel SF, Ramirez SH, Tierney RT, Langford D: Blood biomarkers for brain injury: What are we measuring? Neurosci Biobehav Rev 68:460-473, 2016

20. Kilbaugh TJ, Karlsson M, Byro M, Bebee A, Ralston J, Sullivan $S$, et al: Mitochondrial bioenergetic alterations after focal traumatic brain injury in the immature brain. Exp Neurol 271:136-144, 2015

21. Knöller E, Stenzel T, Broeskamp F, Hornung R, Scheuerle A, McCook O, et al: Effects of hyperoxia and mild therapeutic hypothermia during resuscitation from porcine hemorrhagic shock. Crit Care Med 44:e264-e277, 2016

22. Korley FK, Nikolian VC, Williams AM, Dennahy IS, Weykamp M, Alam HB: Valproic acid treatment decreases serum glial fibrillary acidic protein and neurofilament light chain levels in swine subjected to traumatic brain injury. $\mathbf{J}$ Neurotrauma 35:1185-1191, 2018

23. Lee JJ, Segar DJ, Morrison JF, Mangham WM, Lee S, 
Asaad WF: Subdural hematoma as a major determinant of short-term outcomes in traumatic brain injury. J Neurosurg 128:236-249, 2018

24. Lewis LM, Schloemann DT, Papa L, Fucetola RP, Bazarian J, Lindburg M, et al: Utility of serum biomarkers in the diagnosis and stratification of mild traumatic brain injury. Acad Emerg Med 24:710-720, 2017

25. Lilley E, Armstrong R, Clark N, Gray P, Hawkins P, Mason $\mathrm{K}$, et al: Refinement of animal models of sepsis and septic shock. Shock 43:304-316, 2015

26. Lou Z, Wang AP, Duan XM, Hu GH, Song GL, Zuo ML, et al: Upregulation of NOX 2 and NOX4 mediated by TGF- $\beta$ signaling pathway exacerbates cerebral ischemia/reperfusion oxidative stress injury. Cell Physiol Biochem 46:2103-2113, 2018

27. Mader MM, Leidorf A, Hecker A, Heimann A, Mayr PSM, Kempski O, et al: Evaluation of a new multiparameter brain probe for simultaneous measurement of brain tissue oxygenation, cerebral blood flow, intracranial pressure, and brain temperature in a porcine model. Neurocrit Care 29:291-301, 2018

28. Malhotra AK, Schweitzer JB, Fox JL, Fabian TC, Proctor KG: Cerebral perfusion pressure directed therapy following traumatic brain injury and hypotension in swine. J Neurotrauma 20:827-839, 2003

29. Manley GT, Rosenthal G, Lam M, Morabito D, Yan D, Derugin N, et al: Controlled cortical impact in swine: pathophysiology and biomechanics. J Neurotrauma 23:128-139, 2006

30. Meissner A, Timaru-Kast R, Heimann A, Hoelper B, Kempski O, Alessandri B: Effects of a small acute subdural hematoma following traumatic brain injury on neuromonitoring, brain swelling and histology in pigs. Eur Surg Res 47:141153,2011

31. Nichols TC, Bellinger DA, Merricks EP, Raymer RA, Kloos MT, Defriess N, et al: Porcine and canine von Willebrand factor and von Willebrand disease: hemostasis, thrombosis, and atherosclerosis studies. Thrombosis 2010:461238, 2010

32. Papa L, Brophy GM, Welch RD, Lewis LM, Braga CF, Tan $\mathrm{CN}$, et al: Time course and diagnostic accuracy of glial and neuronal blood biomarkers GFAP and UCH-L1 in a large cohort of trauma patients with and without mild traumatic brain injury. JAMA Neurol 73:551-560, 2016

33. Papa L, Robicsek SA, Brophy GM, Wang KKW, Hannay HJ, Heaton S, et al: Temporal profile of microtubule-associated protein 2: a novel indicator of diffuse brain injury severity and early mortality after brain trauma. J Neurotrauma 35:32-40, 2018

34. Park D, Joo SS, Lee HJ, Choi KC, Kim SU, Kim YB: Microtubule-associated protein 2, an early blood marker of ischemic brain injury. J Neurosci Res 90:461-467, 2012

35. Platt SR, Radaelli ST, McDonnell JJ: The prognostic value of the modified Glasgow Coma Scale in head trauma in dogs. J Vet Intern Med 15:581-584, 2001

36. Purins K, Enblad P, Wiklund L, Lewén A: Brain tissue oxygenation and cerebral perfusion pressure thresholds of ischemia in a standardized pig brain death model. Neurocrit Care 16:462-469, 2012

37. Rimpiläinen J, Pokela M, Kiviluoma K, Anttila V, Vainionpää V, Hirvonen J, et al: Leukocyte filtration improves brain protection after a prolonged period of hypothermic circulatory arrest: A study in a chronic porcine model. J Thorac Cardiovasc Surg 120:1131-1141, 2000
38. Shaver EG, Duhaime AC, Curtis M, Gennarelli LM, Barrett $\mathrm{R}$ : Experimental acute subdural hematoma in infant piglets. Pediatr Neurosurg 25:123-129, 1996

39. Sorby-Adams AJ, Vink R, Turner RJ: Large animal models of stroke and traumatic brain injury as translational tools. Am J Physiol Regul Integr Comp Physiol 315:R165-R190, 2018

40. Talley Watts L, Shen Q, Deng S, Chemello J, Duong TQ: Manganese-enhanced magnetic resonance imaging of traumatic brain injury. J Neurotrauma 32:1001-1010, 2015

41. Timaru-Kast R, Meissner A, Heimann A, Hoelper B, Kempski O, Alessandri B: Acute subdural hematoma in pigs: role of volume on multiparametric neuromonitoring and histology. J Neurotrauma 25:1107-1119, 2008

42. Valadka AB, Gopinath SP, Contant CF, Uzura M, Robertson CS: Relationship of brain tissue $\mathrm{PO}_{2}$ to outcome after severe head injury. Crit Care Med 26:1576-1581, 1998

43. Van den Heuvel C, Blumbergs PC, Finnie JW, Manavis J, Jones NR, Reilly PL, et al: Upregulation of amyloid precursor protein messenger RNA in response to traumatic brain injury: an ovine head impact model. Exp Neurol 159:441-450, 1999

44. Won SY, Dubinski D, Brawanski N, Strzelczyk A, Seifert V, Freiman TM, et al: Significant increase in acute subdural hematoma in octo- and nonagenarians: surgical treatment, functional outcome, and predictors in this patient cohort. Neurosurg Focus 43(5):E10, 2017

45. Xiong Y, Mahmood A, Chopp M: Animal models of traumatic brain injury. Nat Rev Neurosci 14:128-142, 2013

\section{Disclosures}

The authors report no conflict of interest concerning the materials or methods used in this study or the findings specified in this paper.

\section{Author Contributions}

Conception and design: Kapapa, Radermacher. Acquisition of data: Datzmann, Kapapa, Merz, Unmuth, Hoffmann, Mathieu, Mayer, Mauer, Röhrer, Möller, Nussbaum, Calzia, Gröger, Radermacher, Wepler. Analysis and interpretation of data: Datzmann, Kapapa, Scheuerle, McCook, Merz, Unmuth, Mathieu, Mauer, Yilmazer-Hanke, Möller, Nussbaum, Calzia, Gröger, Hartmann, Radermacher, Wepler. Drafting the article: Datzmann, Radermacher. Critically revising the article: Datzmann, Kapapa, Scheuerle, McCook, Unmuth, Hoffmann, Mayer, Mauer, Röhrer, Möller, Calzia, Radermacher, Wepler. Reviewed submitted version of manuscript: Datzmann, Kapapa, Yilmazer-Hanke, Möller, Calzia, Radermacher, Wepler. Approved the final version of the manuscript on behalf of all authors: Datzmann. Statistical analysis: Datzmann, Radermacher, Wepler. Administrative/ technical/material support: Datzmann, Kapapa, Scheuerle, McCook, Merz, Hoffmann, Mathieu, Mayer, Mauer, Röhrer, Yilmazer-Hanke, Nussbaum, Calzia, Gröger, Hartmann. Study supervision: Radermacher.

\section{Correspondence}

Thomas Datzmann: University Hospital Ulm, Germany. thomas. datzmann@uni-ulm.de. 